Herbert Silverstein, M.D., Horace Norrell, M.D., Hayes Wanamaker, M.D., and John Flanzer, M.D.

\title{
MICROSURGICAL POSTERIOR
} FOSSA VESTIBULAR

NEURECTOMY: AN EVOLUTION IN TECHNIQUE

\begin{abstract}
Between 1925 and 1945, Walter Dandy and Kenneth McKenzie performed more than 700 posterior fossa eighth nerve sections and vestibular neurectomies, treating the intractable vertigo accompanying Meniere's disease. During the past 10 years, using microsurgical techniques and reaching the posterior fossa through the temporal bone, vestibular neurectomy has enjoyed a resurgence of popularity. When hearing is to be preserved, vestibular neurectomy is the surgical treatment of choice, if the patient fails to undergo a remission of the vertigo of Meniere's disease. This report reviews 115 consecutive vestibular neurectomies performed for the treatment of Meniere's disease from 1978 to 1988

In 1978, the retrolabyrinthine vestibular neurectomy (RVN) was introduced; a procedure in which the posterior fossa is entered anterior to the sigmoid sinus and behind the labyrinth. During the last three years, the approach to the posterior fossa has been a small dural opening behind the sigmoid sinus, the combined retrolabyrinthine-retrosigmoid (R-R) approach. There have been no cases of facial paralysis and no serious complications. A high incidence of headache $(75 \%)$ resulted when the posterior wall of the internal auditory canal was drilled away for better exposure. Transient cerebrospinal fluid (CSF) leaks occurred in 7\% of the patients having the RVN; the incidence was $3 \%$ when the combined R-R approach was used. In the RVN series, wound infection occurred in $20 \%$ of cases until perioperative antibiotics reduced the rate to $3 \%$. The results in curing or improving vertigo have been excellent $(94 \%)$, and hearing has been preserved to within $20 \mathrm{~dB}$ of the preoperative levels in $76 \%$. Until a cure for Meniere's disease is found, microsurgical posterior fossa vestibular neurectomy remains the best treatment.
\end{abstract}

Today, vestibular neurectomy is accepted as the procedure of choice to preserve hearing and relieve intractable vertigo associated with unilateral vestibular disorders that are refractory to medical management. Frazier ${ }^{1}$ in 1904 was the first to perform an eighth nerve section through the posterior fossa to relieve symptoms of vertigo in Meniere's disease. Twenty-one years later in 1925, Dandy ${ }^{2}$ began performing eighth nerve sections. Cutting only the superior one half of the eighth nerve (vestibular fibers) was introduced by McKenzie ${ }^{3}$ in 1931. In 1932, Dandy performed the first of his 624 vestibular neurec- tomies, the largest series in the world. ${ }^{4}$ After his death, peripheral destructive procedures of the labyrinth became popular and have remained so until recently; unfortunately, hearing is lost with the surgery. The first microsurgical vestibular neurectomy was performed by William House $^{5}$ in 1961, through a subtemporal approach. In this procedure, the roof of the internal auditory canal (IAC) is drilled away and the vestibular nerve transected. Because of the technical difficulty and associated complications, few middle fossa nerve sections were performed in the United States. 
In 1978 , while removing a ninth nerve neurilemmoma from the posterior fossa through a transmastoid retrolabyrinthine approach, a clear cleavage plane was discovered in the eighth nerve between the cochlear and vestibular nerves. ${ }^{6}$ Gross and microscopic laboratory studies confirmed that vestibular neurectomy could be performed routinely through a retrolabyrinthine approach. This report presents the indications, anatomic findings, and evolution of the microsurgical approach to the posterior fossa for vestibular neurectomy.

\section{INDICATIONS AND CONTRAINDICATIONS}

Although classic unilateral Meniere's disease is the most common indication, vestibular neurectomy can be useful for other labyrinthine disorders (such as traumatic labyrinthitis). Patient's choice is a strong consideration in the decision of when to operate. Some patients may have one or two severe Meniere's attacks a month and not have their life-styles sufficiently affected to warrant a major surgical procedure to correct the problem. Other patients with only two or three attacks a year can be so severely affected that they live in constant dread of the next recurrence. Before surgery can be considered, objective evidence of unilateral inner ear disease should be provided by audiogram, electronystagmography, and electrocochleography.

Contraindications to vestibular neurectomy include bilateral vestibular disease, poor medical condition, ataxia or other indications of possible significant central nervous system involvement, and vertigo arising from an onlyhearing ear. Vertigo from an ear with very poor hearing (80 dB speech reception threshold [SRT] and less than $20 \%$ discrimination) is usually more appropriately treated with a transcochlear eighth nerve section. ${ }^{7}$

A previous endolymphatic sac operation is not a contraindication, nor is old age when the patient is healthy and has good balance function. Vestibular neurectomy has been done successfully in patients over 70 years old with excellent results and little additional morbidity. Elderly persons usually take longer to regain good balance function postoperatively than do younger individuals.

\section{ANATOMY: VESTIBULAR, COCHLEAR, AND FACIAL NERVES}

The anatomy is described as if the patient were supine and the head turned away from the surgeon (Fig. 1). At the labyrinthine end of the IAC, six separate branches of the seventh and eighth cranial nerves enter the temporal bone: the facial nerve, nervus intermedius (NI), superior vestibular nerve, saccular nerve, posterior ampullary nerve, and cochlear nerve. The transverse (falciform) crest, which lies in a perpendicular plane, divides the lateral IAC into superior and inferior compartments. A bridge of bone (Bill's bar) separates the superior half of the IAC into an anterior-superior quadrant containing the facial nerve and nervus intermedius, and a posterior-superior quadrant containing the superior vestibular nerve. Anteriorly and inferior to the falciform crest lies the cochlear nerve, hidden from the surgeon by the inferior vestibular nerve. The posterior ampullary nerve lies in a separate canal (the singular canal) that enters the IAC in the posterior-inferior quadrant, approximately $2 \mathrm{~mm}$ medial to the falciform crest. This reliable landmark is the point at which drilling stops when the posterior wall of the IAC is being surgically removed. Thin section computed tomography of the labyrinth is routinely obtained to visualize the exact location of the singular canal. The inferior vestibular nerve is formed when the saccular nerve joins the posterior ampullary nerve just medial to the falciform crest.

The superior vestibular nerve innervates the superior semicircular canal, horizontal semicircular canal, saccule, and utricle. The inferior vestibular nerve innervates the saccule (saccular nerve) and the posterior semicircular canal (posterior ampullary nerve).

The separation between the cochlear and vestibular nerves, the cochleovestibular (CV) cleavage plane at the labyrinthine end of the IAC, lies in the superior-inferior plane, with the vestibular nerves occupying the posterior half of the IAC. Between the falciform crest and the porus acousticus, the superior and inferior vestibular nerves fuse. Laterally, within the IAC is a constant, well-delineated cleavage between these two nerves. 8

At the distal end of the IAC, the cochlear nerve lies anterior to the inferior vestibular nerve. The cochlear and inferior vestibular nerves fuse within the IAC, just medial to the falciform crest. The cochlear and vestibular nerves then rotate $90^{\circ}$, so that the cochlear nerve, which at first lies anterior to the inferior vestibular nerve, rotates to lie caudal and inferior to the vestibular nerve as it enters the porus acousticus. ${ }^{8}$ From a medial vantage point, the rotation in the left ear is clockwise; in the right ear it is counterclockwise. Most of the rotation occurs within the IAC; only slight rotation occurs in the cerebellopontine (CP) angle (Fig. 2). The cochlear nerve leaves the brainstem caudal and slightly dorsal to the vestibular nerve. The flocculus of the cerebellum covers $5 \mathrm{~mm}$ of the eighth cranial nerve at the brainstem. The $90^{\circ}$ rotation of the cochlear and vestibular nerves is not recognized in modern neurosurgical publications. ${ }^{9}$

After the vestibular and cochlear nerves fuse within the IAC, the CV cleavage plane usually persists grossly and histologically. The vestibular fibers remain segregated and are cephalad or superior; the cochlear fibers are caudal or inferior. Occasionally, inferior vestibular fibers will run with the cochlear nerve, while the efferent cochlear fibers run in the inferior vestibular nerve. Near the labyrinth, the CV cleavage plane runs in a superiorinferior direction and, because of rotation, in an anterior- 


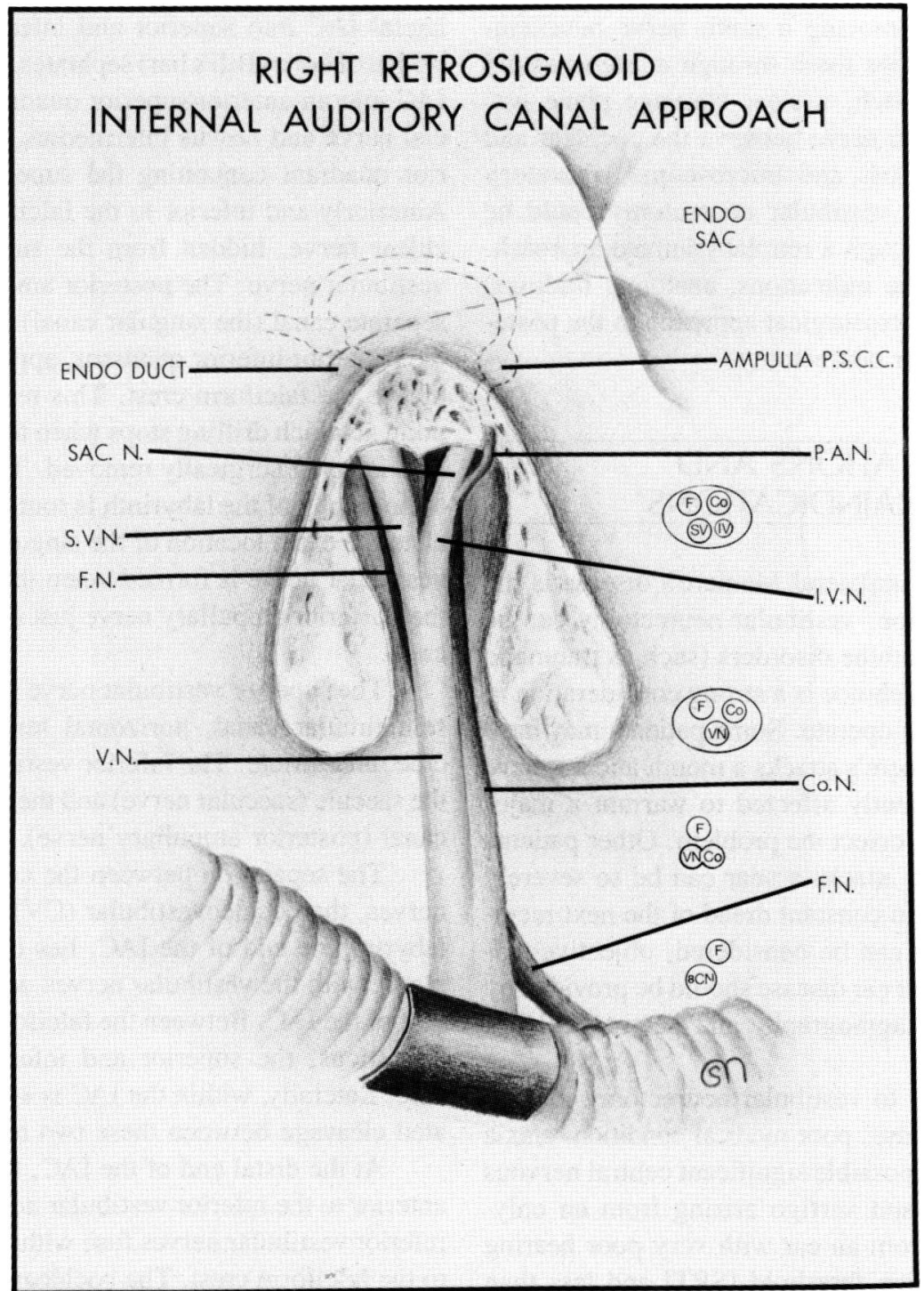

Figure 1. Anatomy of the seventh and eighth cranial nerves as seen from otologic surgical position. Note $90^{\circ}$ rotation of cochlear and vestibular nerves. ENDO SAC: endolymphatic sac; SAC. N.: saccular nerve; P.S.C.C.: posterior semicircular canal; P.A.N.: posterior ampullary nerve; S.V.N.: superior vestibular nerve; I.V.N.: inferior vestibular nerve; F.N.: facial nerve; V.N.: vestibular nerve; Co.N.: cochlear nerve; $8 \mathrm{CN}$ : 8th cranial nerve; $F$ : facial nerve.

posterior direction in the $\mathrm{CP}$ angle. In the $\mathrm{CP}$ angle, the $\mathrm{CV}$ cleavage plane appears grossly as a fine septum along the eighth cranial nerve in $75 \%$ of patients.

In the lateral IAC, the facial nerve is positioned in the anterior-superior quadrant, anterior to the superior vestibular nerve, running to a ventral-caudal position as it exits the brainstem. The facial nerve remains ventrally positioned and hidden by the eighth cranial nerve along much of its entire course. In the IAC, the facial nerve is connected to the superior vestibular nerve by the Rasmussen vestibulo-facial anastomosing fibers, and in the $\mathrm{CP}$ angle, the facial nerve lies adjacent to, but distinct from, the eighth nerve. Although it remains hidden from the surgeon's view by the eighth cranial nerve, the facial nerve can easily be visualized by gentle retraction of the superior vestibular nerve in the IAC or the eighth nerve in the $\mathrm{CP}$ angle. The facial nerve exits the brainstem $3 \mathrm{~mm}$ ventral and usually caudal to the eighth nerve root entry zone. In the IAC, the seventh nerve appears whiter than the eighth nerve, in the $\mathrm{CP}$ angle it appears more gray.

The NI, which may consist of a single nerve or multiple bundles, runs between the seventh and eighth nerves through their entire course. The NI enters the brainstem closest to the eighth nerve and usually delineates the CV cleavage plane on the anterior surface of the eighth nerve. 


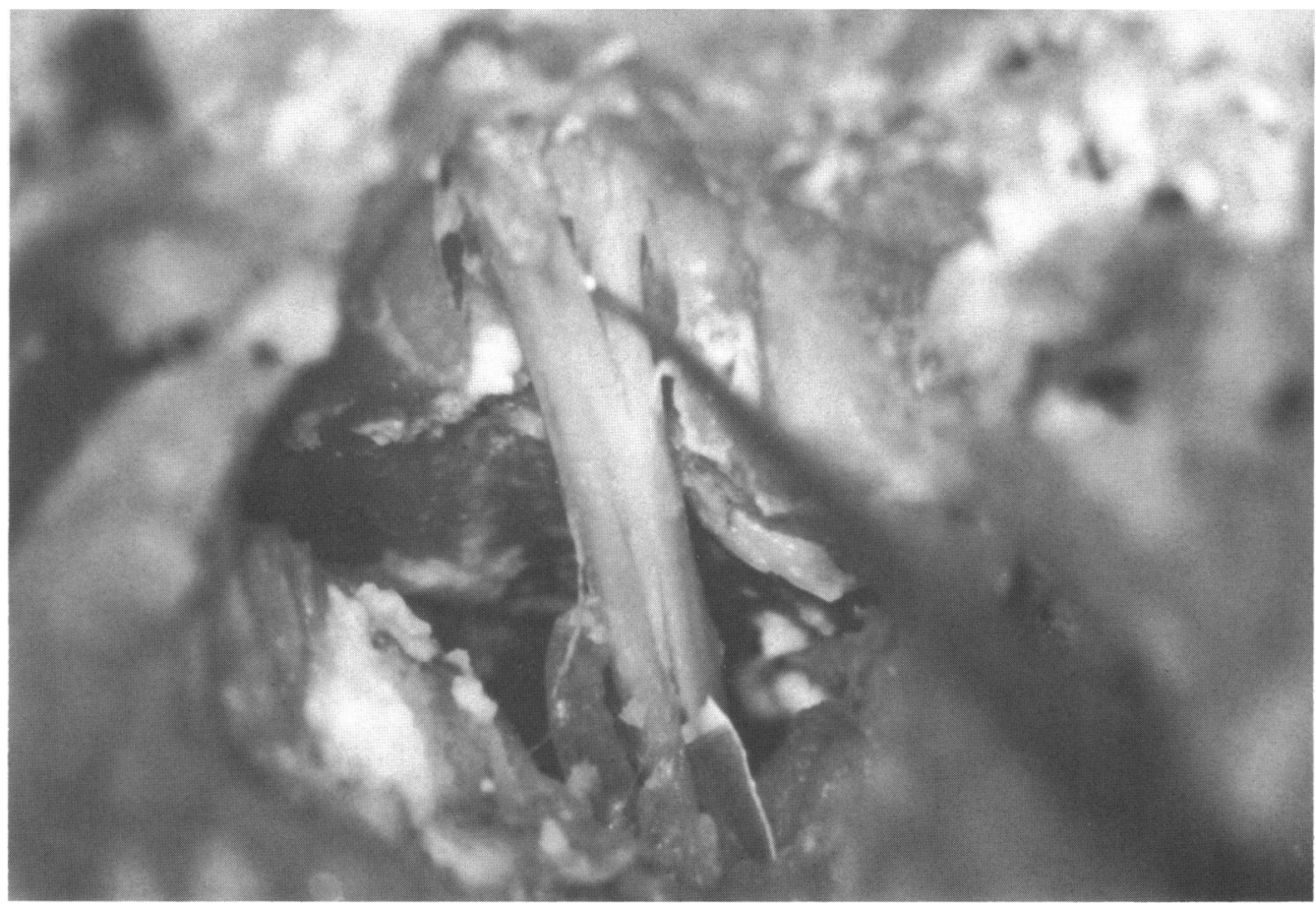

Figure 2. Cadaver specimen of right eighth cranial nerve. Note $90^{\circ}$ rotation of cochlear and vestibular nerves. At the bottom of the figure, a triangular marker is inserted in the cochleovestibular cleavage plane. The posterior portion of the eighth cranial nerve is shown. The anterior portion faces away from the surgeon.

\section{OPERATIVE APPROACHES FOR VESTIBULAR NEURECTOMY}

\section{Middle Fossa Vestibular Neurectomy}

From 1963 to 1978, the middle fossa (subtemporal) approach exclusively was used to section the vestibular nerve. Results for vertigo relief were good, but the procedure was formidable, anatomic landmarks were unreliable, and complications, such as facial nerve weakness or deafness, did occur. ${ }^{10}$ Patients over 60 years of age were generally not candidates. The procedure was technically difficult and carried a high risk of complications; thus, many patients, who had significant disability, were not enthusiastically offered a middle fossa vestibular neurectomy.

\section{Posterior Fossa Vestibular Neurectomy}

\section{Retrolabyrinthine Approach}

In 1978, the senior author developed the retrolabyrinthine approach to the posterior cranial fossa for vestibular neurectomy (RVN).6,11 Although previously used as an approach for trigeminal nerve section, 12 its application for selective vestibular nerve section had not been described.

A simple mastoidectomy is performed, and the lateral sinus and posterior fossa dura just behind the sinus and in front of it are exposed. The endolymphatic sac is widely exposed and the bone overlying the posterior semicircular canal identified. The sigmoid sinus is collapsed with a retractor, and the dura incised in a $\mathrm{C}$-shape anterior to the sigmoid sinus based on the labyrinth (Fig. 3). Intravenous mannitol $(1.5 \mathrm{gm} / \mathrm{kg}$ to a maximum of 100 $\mathrm{gm})$ is given when the drilling begins, which allows a wider exposure of the $\mathrm{CP}$ angle during surgery. Using the Nicolet Pathfinder (Nicolet, Madison, WI), auditory responses are monitored from the brainstem, cochlea, and eighth cranial nerve. Facial nerve monitoring is performed with the WR-S8 Stimulator/Monitor ${ }^{13,14}$ (WR Medical, Stillwater, MN).

Intraoperative monitoring of auditory-evoked responses was found to enhance the surgeon's ability to preserve hearing and allows the surgeon to inform the family immediately after surgery that hearing will probably be unchanged. ${ }^{15} \mathrm{After}$ the cleavage plane is visualized under high-power magnification, a longitudinal incision is made in the cleavage plane, the cochlear and vestibular fibers are separated, and the vestibular nerve is transected. Several landmarks are helpful in finding the cleavage plane. The vestibular nerve often appears grayer, and the cochlear nerve whiter; the cochlear fibers are more numerous, averaging 31,000 , whereas the vestibular fibers average 18,000 . A fine blood vessel frequently courses on the surface between the cochlear and vestibular fibers. A mirror can be used to view the anterior surface of the eighth nerve, since the cleavage plane is sometimes more visible from this surface. The NI, which usually lies in the 


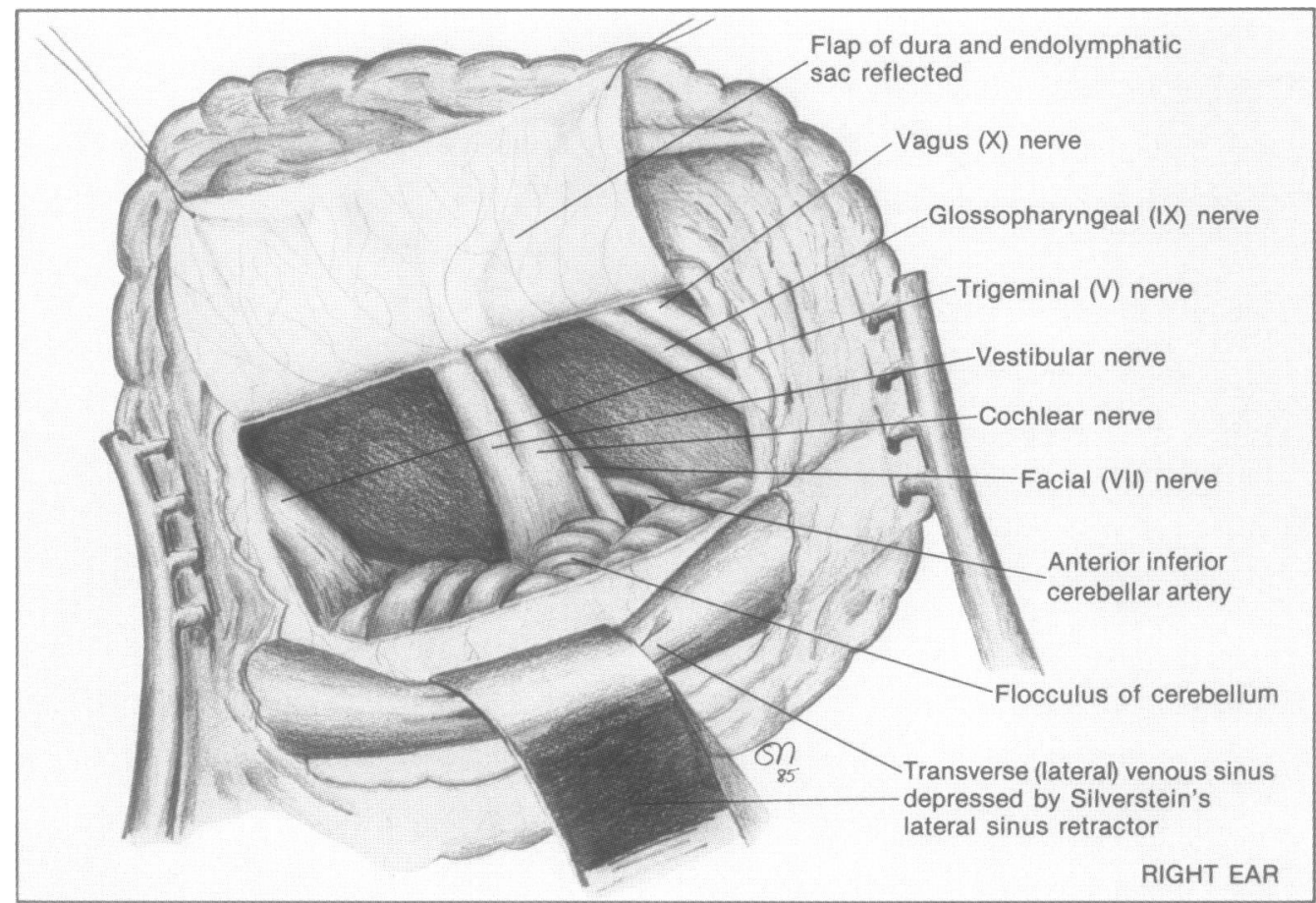

Figure 3. Retrolabyrinthine exposure of the cranial nerves. Vestibular nerve has not been transected as yet.

cleavage plane, can also be seen anteriorly. The superior one half of the eighth cranial nerve is transected when a cleavage plane cannot be readily identified. Most vestibular fibers will be cut, and most cochlear fibers will be spared using this technique. The dura is closed with three or four interrupted 4-0 silk sutures and the mastoid cavity is filled with abdominal adipose tissue.

\section{Retrosigmoid_Internal Auditory Canal Approach}

In 1985, in an attempt to perform a more complete vestibular neurectomy near the labyrinth, to improve vertigo relief and hearing preservation and to decrease the incidence of cerebrospinal fluid (CSF) leak, the retrosigmoid (RSG) IAC approach was used. Because the cleavage plane between cochlear and vestibular fibers is more completely developed within the IAC, a more complete and selective vestibular neurectomy can be performed by cutting the nerve within the IAC.

In this procedure, a posterior fossa craniotomy is performed immediately behind the lateral sinus, and the cerebellum is retracted to give exposure to the seventh and eighth cranial nerves and the IAC. The posterior wall of the IAC is removed to the singular canal, thereby exposing the branches of the eighth cranial nerve. The superior vestibular nerve and the singular nerve (posterior ampullary nerve) are sectioned. The inferior vestibular fibers that innervate the saccule are not divided because of their close association with cochlear fibers. Since the saccule has no known vestibular function in man, sparing these fibers does not result in postoperative vertigo attacks.

This procedure offers several advantages over the RVN. No abdominal fat is needed to fill the defect, thus the procedure can be performed on thin patients. Since the exposure does not enter the mastoid, patients who have had chronic mastoiditis, a sclerotic mastoid, or an anterior-lying sigmoid sinus can be candidates for the RSGIAC approach. ${ }^{16,17}$

\section{Combined Retrolabyrinthine-Retrosigmoid Approach}

A further evolution of the vestibular neurectomy procedure, the combined retrolabyrinthine-retrosigmoid (R-R) vestibular neurectomy occurred in 1987.18 The combined R-R incorporates the advantages of the RVN and RSG-IAC approaches. It also allows the surgeon to assess the CV cleavage plane in the posterior fossa and decide where the neurectomy should be performed. If a good CV cleavage plane exists, the vestibular nerve section will be done in the $\mathrm{CP}$ angle. If not, the IAC can be opened and the superior vestibular and posterior ampullary nerves sectioned within the IAC. In this approach, a limited mastoidectomy is done. The lateral venous sinus (LVS) is exposed from the transverse sinus inferiorly 3 $\mathrm{cm}$, and the posterior fossa dura is exposed for $1.0 \mathrm{~cm}$ posterior to the LVS. The dural incision is made $3 \mathrm{~mm}$ behind and parallel to the LVS for $2.5 \mathrm{~cm}$ (Fig. 4). The LVS is retracted anteriorly using stay sutures placed in the dural cuff. After the arachnoid cistern is opened, the eighth nerve is examined and, if a cleavage plane is present, the vestibular nerve section is performed in the $\mathrm{CP}$ angle, as in the retrolabyrinthine approach (Fig. 5). If no cleavage plane is identified, then the dura is reflected off the temporal bone, the IAC is opened with a diamond burr, and the superior vestibular and posterior ampullary nerves are divided, as in the retrosigmoid approach (Fig. 


\section{COMBINED RETROLAB-RETROSIGMOID VESTIBULAR NEURECTOMY}

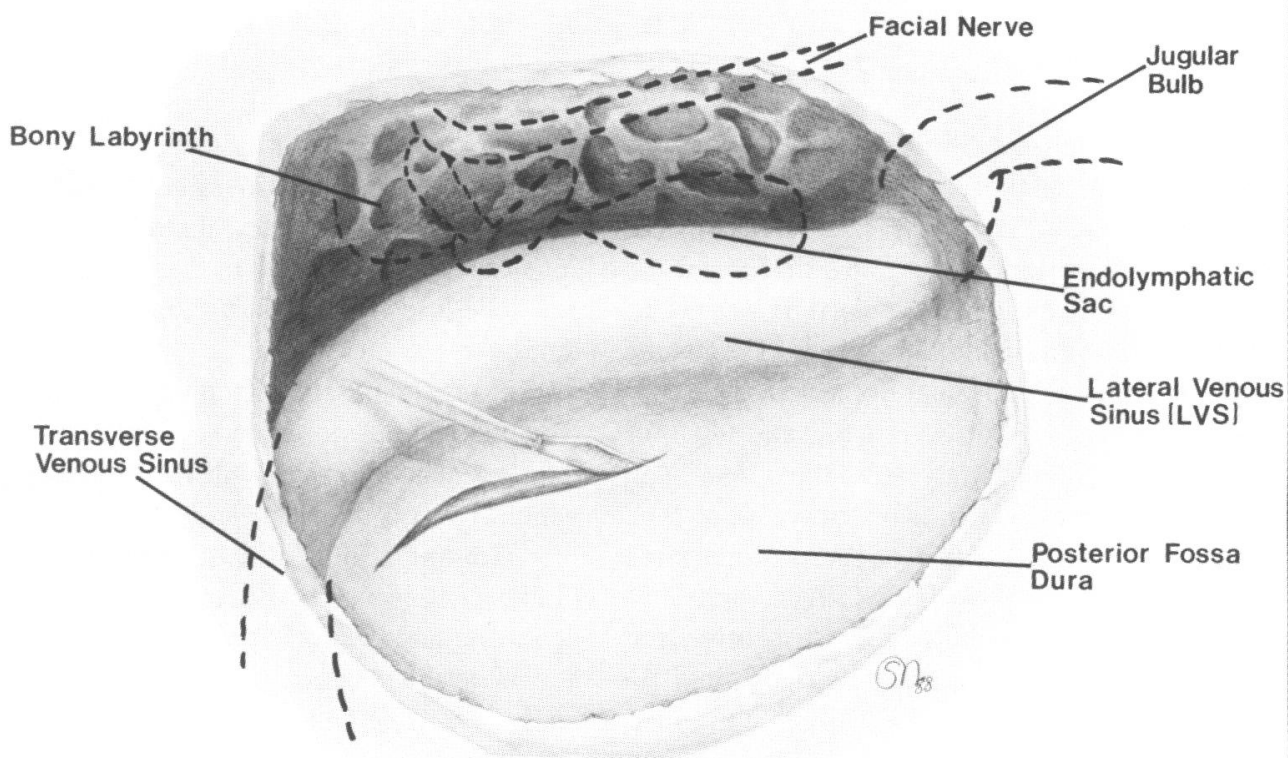

RIGHT EAR

Figure 4. Combined retrolabrynthine-retrosigmoid vestibular neurectomy. Dural incision is made behind sigmoid sinus.

\section{GOOD C V CLEAVAGE IN CPA VESTIBULAR NEURECTOMY WITHIN CPA}

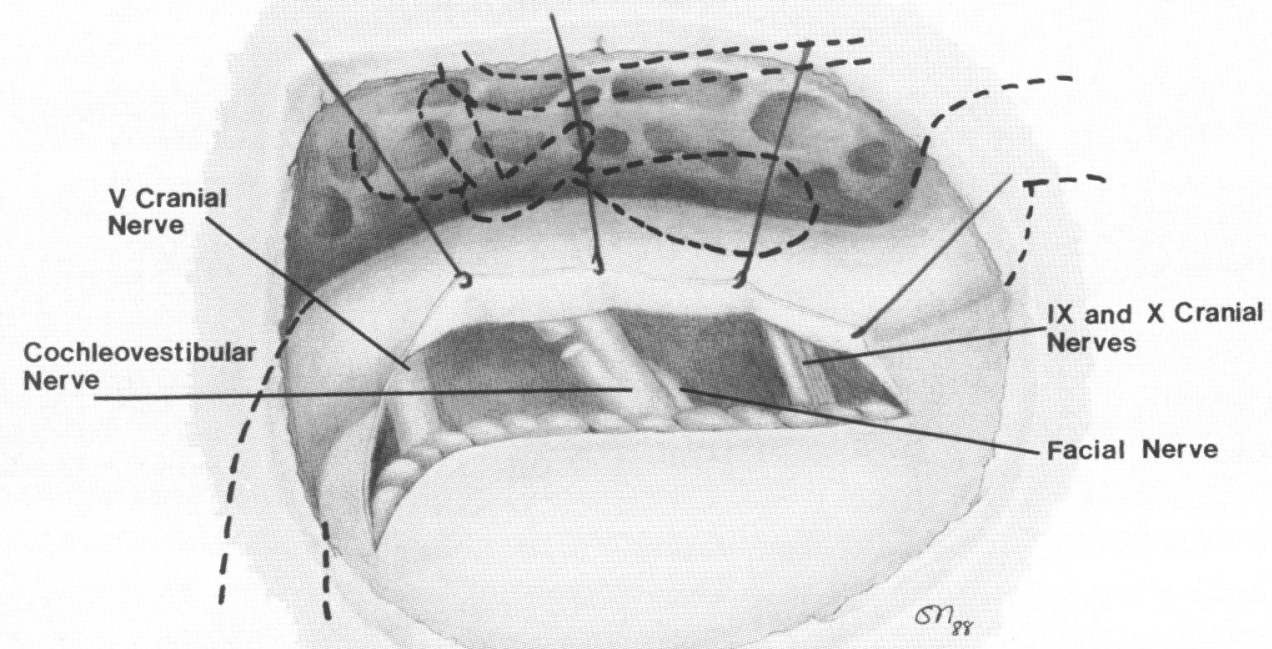

RIGHT EAR

Figure 5. Exposure seen from right combined R-R approach. Patient had good cochleovestibular (CV) plane and vestibular neurectomy was done in posterior fossa. CPA: cerebellopontine angle. 
6). The mastoid air cells are sealed with bone wax, the dura is closed in a watertight fashion, and the surgical defect is filled with abdominal adipose tissue.

\section{RESULTS}

One hundred fifteen vestibular neurectomies for Meniere's disease have been performed: 67 RVN, 15 RSGIAC, and 33 combined R-R. Follow-up has been at least 2 years. No patient has developed Meniere's disease in the opposite ear. The overall cure rate for vertigo was $84 \%$, with an additional $10 \%$ significantly improved. The results have been similar with each surgical approach. The cure rate was $82 \%$ for RVN, $87 \%$ for RSG-IAC, and $93 \%$ for combined R-R (Table 1). Hearing results have also been good with each approach. The postoperative pure-tone average has remained within $20 \mathrm{~dB}$ of the postoperative level in $71 \%$ with RVN, $67 \%$ with RSG-IAC, and $91 \%$ with combined R-R, $76 \%$ overall. The discrimination score has remained within $20 \%$ postoperatively; in $70 \%$ with RVN, $47 \%$ with RSG-IAC, and $82 \%$ with combined R-R, $69 \%$ overall (Table 2).

Complications encountered in RVN are listed in Table 3. CSF leaks occurred in $7 \%$ because a tight closure of the posterior fossa dura was not possible. CSF leaks, if present, are managed with the placement of a continuous lumbar drain for 2 to 5 days to control CSF pressure. Lumbar drainage has been used for the past 8 years, and no patient has required a second operation to eliminate a CSF leak. Wound infection rate has been drastically reduced since we began using perioperative antibiotics. No antibiotics were used in the first 40 cases of RVN, and the infection rate was $20 \%$. All infections were superficial and responded rapidly to local treatment and oral antibiotics, but this rate was regarded as unacceptably high. The use of intravenous nafcillin in a three-dose regimen ( $2 \mathrm{gm}$ preoperatively, 2 gm intraoperatively, and 2 gm 6 hours later) has reduced the infection rate to $3 \%$. In cases of penicillin sensitivity, clindamycin $600 \mathrm{gm}$ is used in a similar dosage schedule. No cases of meningitis have been encountered.

No facial paralysis or death has occurred. Postoperative unsteadiness is expected (as seen after labyrinthectomy), but it improves with central compensation and is generally well tolerated and accepted.

Complications of RSG-IAC have been infrequent (Table 3); however, severe chronic persistent postoperative headache has been a problem. In $50 \%$ of the patients the headaches have been difficult to control with non-narcotic analgesics. The headaches appear to decrease in severity in a 2 -year period. Four of the 15 patients (27\%) still have severe headaches requiring continuous medication after 2 years. The headache is usually unilateral (ipsilateral to surgery), most severe in the temporal region, and frequently retro-orbital. The cause for the headache has remained a mystery, particularly since this approach has been used successfully for fifth nerve section or vascular

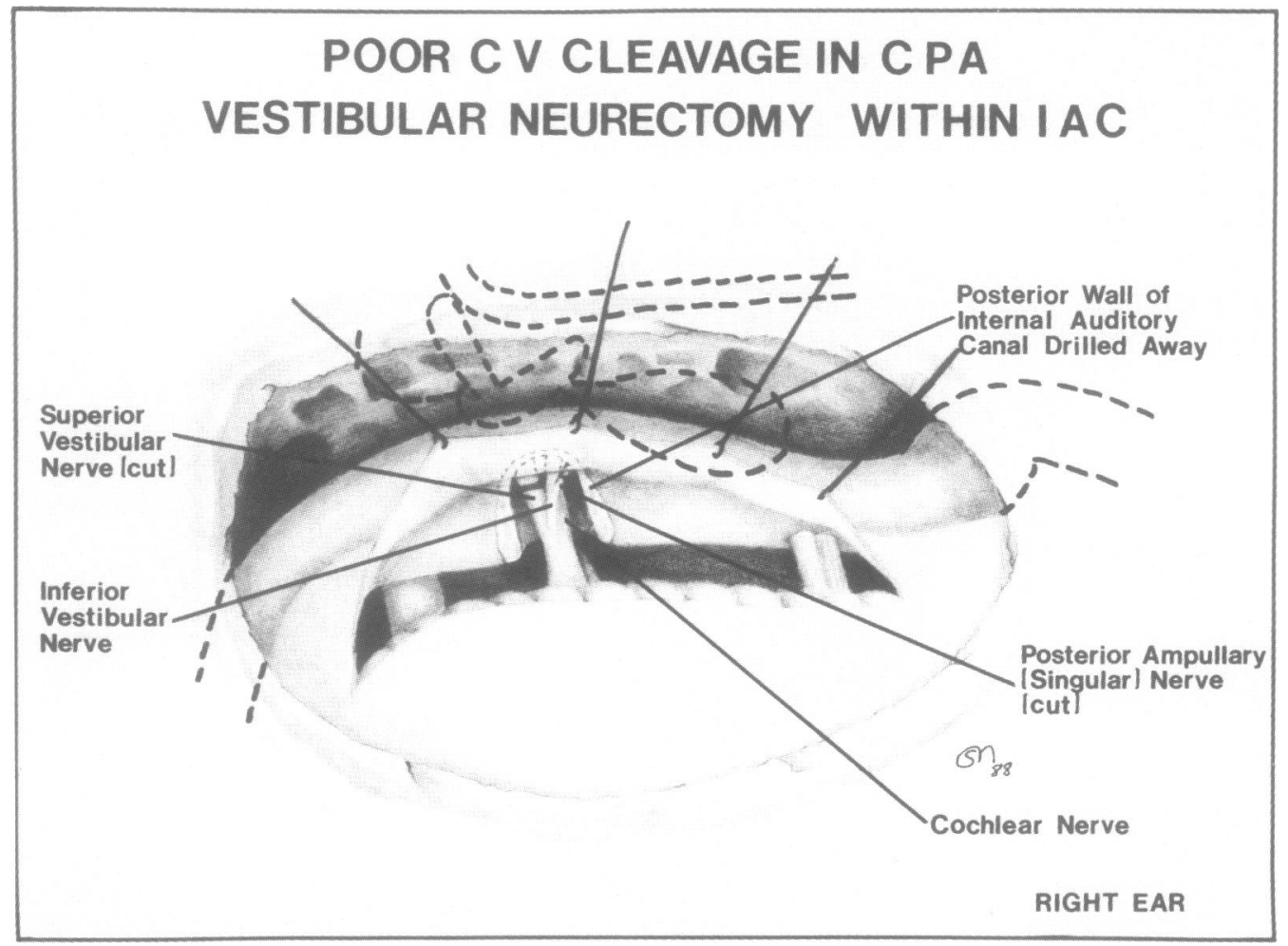

Figure 6. Combined R-R approach. Poor cochleovestibular (CV) cleavage plane and vestibular neurectomy was performed after removing bone from IAC. CPA: cerebellopontine angle. 
Table 1. Vertigo Results*

\begin{tabular}{lcccc}
\hline & $\begin{array}{c}R V N \\
(n=67)\end{array}$ & $\begin{array}{c}R S G-I A C \\
(n=15)\end{array}$ & $\begin{array}{c}\text { Combined R-R } \\
(n=14)\end{array}$ & $\begin{array}{c}\text { Overall } \\
(n=96)\end{array}$ \\
\hline Cured (\%) & 82 & 87 & 93 & 84 \\
Improved (\%) & 10 & 13 & 7 & 10 \\
Unchanged (\%) & 8 & 0 & 0 & 6 \\
\hline
\end{tabular}

*Follow-up was 2 years or greater.

Table 2. Hearing Results*

\begin{tabular}{lcccc}
\hline & $\begin{array}{c}R V N \\
(n=67)\end{array}$ & $\begin{array}{c}R S G-I A C \\
(n=15)\end{array}$ & $\begin{array}{c}\text { Combined R-R } \\
(n=22)\end{array}$ & $\begin{array}{c}\text { Overall } \\
(n=104)\end{array}$ \\
\hline Pure-tone average within 20 dB (\%) & 71 & 67 & 91 & 76 \\
Discrimination within 20\% (\%) & 70 & 47 & 82 & 69 \\
\hline
\end{tabular}

*Follow-up was 2 years or greater.

Table 3. Complications

\begin{tabular}{lccc}
\hline & $\begin{array}{c}R V N \\
(n=67)\end{array}$ & $\begin{array}{c}R S G-I A C \\
(n=14)\end{array}$ & $\begin{array}{c}\text { Combined R-R } \\
(n=33)\end{array}$ \\
\hline CSF leak (\%) & 7 & 0 & 3 \\
Wound infection (\%) & $3 *$ & 0 & 3 \\
Meningitis (\%) & 0 & 0 & 0 \\
Facial paralysis (\%) & 0 & 0 & 0 \\
Significant headaches (\%) & 0 & $50^{+}$ & 9 \\
\hline
\end{tabular}

*Wound infection $20 \%$ before perioperative antibiotics.

tMay be related to drilling IAC.

decompression and is quite similar to the suboccipital approach often used for acoustic neuroma surgery. The postoperative headaches remain a major concern and have tempered our enthusiasm for the classic RSG-IAC vestibular neurectomy.

The complications after combined R-R vestibular neurectomy are listed in Table 3 . The incidence of headache $(9 \%)$ has been greatly reduced using the combined R-R vestibular neurectomy. One patient had a CSF leak with a wound infection and one had a complete sensorineural hearing loss postoperatively. The wound infection rate was $3 \%$.

\section{DISCUSSION}

The original RVN has proved to be an excellent procedure with minimal complications. For several reasons, a better procedure was contemplated: the inability to determine a definite CV cleavage plane in $25 \%$ of cases, $30 \%$ of postoperative patients having a further sensorineural loss and some having a conductive loss, the complications of CSF leaks (7\%) or wound infection (3\%), and the inability to expose the IAC if needed to see a better cleavage plane.

The ideal procedure would be one in which an intraoperative decision could be made as to whether the ves- tibular nerve section should be made in the $\mathrm{CP}$ angle or within the IAC, depending on the presence or absence of a distinct $\mathrm{CV}$ cleavage plane; the hearing would less likely be affected and CSF leaks could be avoided by a better dural closure. The RSG-IAC approach introduced in 1985 appeared to offer these advantages. The superior vestibular nerve could be routinely separated from the facial and inferior vestibular nerves in the IAC, but it was difficult to separate the inferior vestibular nerve from the cochlear nerve near the porus acousticus without causing a sensorineural hearing loss. This problem was solved by dividing the posterior ampullary nerve and leaving the saccular nerve intact. This allowed a complete denervation of the vestibular labyrinth without compromising auditory function. The dura could be closed completely, thus avoiding the CSF leak problem.

The only difference between the standard RVN procedure and the new combined R-R (1987) is that, in the latter, a limited mastoidectomy is done skeletonizing the LVS, and the dural incision is made behind, rather than anterior to, the LVS. Once the CSF is released, the cerebellum falls away from the temporal bone and allows good exposure of the $\mathrm{CP}$ angle, without cerebellar retraction. The distance from the dural opening to the eighth cranial nerve is about $1 \mathrm{~cm}$ farther than in the RVN exposure. The exposure with the combined R-R approach allows the surgeon to remove the posterior wall of the IAC, if needed, 
to section the vestibular nerve. Because of the angle of the approach to the $\mathrm{CP}$ angle, opening the IAC is not possible in the standard RVN approach. The use of stay sutures to elevate and retract the LVS forward allows easier and greater exposure of the $\mathrm{CP}$ angle without cerebellar retraction. Less bone is removed over the cerebellum in the combined procedure than in a RSG-IAC craniotomy. Since most of the mastoid bone is left intact, there is less chance of conductive hearing loss by herniating adipose tissue into the middle ear or bone dust fixing the stapes bone. The combined R-R appears to offer advantages over both the RVN and the RSG-IAC procedures. Since less bone is removed in the combined procedure, the surgical time is reduced. The watertight closure of the dura reduces the chance of a CSF leak found in the RVN. Thus far, the hearing results have been better than those of either the RVN and RSG-IAC. The results in curing vertigo have been excellent ( $93 \%)$. The combined R-R approach represents an evolution in technique and is an improvement over the RVN and RSG-IAC approaches for vestibular neurectomy.

\section{REFERENCES}

1. Frazier $\mathrm{CH}$ : Intracranial division of the auditory nerve for persistent aural vertigo. Surg Gynecol Obstet 15:524-529, 1912

2. Dandy WE: Meniere's disease; its diagnosis and a method of treatment. Arch Surg 16:1127-1152, 1928

3. McKenzie KG: Intracranial division of the vestibular portion of the auditory nerve for Meniere's disease. Can Med Assoc J 34:369_ 381,1936

4. Greene RE: Surgical treatment of vertigo, with follow-up on Walter Dandy's cases. Clin Neurosurg 6:141-152, 1958

5. House WF: Surgical exposure of the internal auditory canal and its contents through the middle cranial fossa. Laryngoscope 71 : $1363-1385,1961$

6. Silverstein H, Norrell H: Retrolabyrinthine surgery: A direct ap- proach to the cerebellopontine angle. Otolaryngol Head Neck Surg 88:462-269, 1980

7. Silverstein H: Transmeatal labyrinthectomy with and without cochleovestibular neurectomy. Laryngoscope 86:1777-1791, 1976

8. Silverstein H: Cochlear and vestibular gross and histologic anatomy (as seen from the postauricular approach). Otolaryngol Head Neck Surg 92:207-211, 1984

9. Silverstein H, Norrell H, Haberkamp T, et al: The unrecognized rotation of the vestibular and cochlear nerves from the labyrinth to the brainstem: Its implications in surgery of the eighth cranial nerve. Otolaryngol Head Neck Surg 95:543-549, 1986

10. Silverstein $\mathrm{H}$, Norrell $\mathrm{H}$, Haberkamp $\mathrm{T}$ : A comparison of retrosigmoid IAC, retrolabyrinthine and middle fossa vestibular neurectomy for treatment of vertigo. Laryngoscope 97:165-173, 1987

11. Silverstein $H$, Norrell $H$ : Retrolabyrinthine vestibular neurectomy. Otolaryngol Head Neck Surg 90:778-782, 1982

12. Hitselberger WE, Pulec JL: Trigeminal nerve (posterior root) retrolabyrinthine selective section. Arch Otolaryngol 96:412415,1972

13. Silverstein $H$, Smouha E, Jones R: Routine intraoperative facial nerve monitoring during otologic surgery. Am J Otol 9:269275,1988

14. Silverstein H, Smouha E, Jones R: Routine identification of the facial nerve using electrical stimulation during otological and neurotological surgery: Laryngoscope 98:726-730, 1988

15. Silverstein H, McDaniel A, Wazen J, Norrell H: Retrolabyrinthine vestibular neurectomy with simultaneous monitoring of 8th nerve and brain stem auditory evoked potentials. Otolaryngol Head Neck Surg 93:736-742, 1985

16. Silverstein H, Norrell H, Smouha E: Retrosigmoid-internal auditory canal approach vs retrolabyrinthine approach for vestibular neurectomy. Otolaryngol Head Neck Surg 97:300-307, 1987

17. Silverstein H, Norrell H, Smouha E, et al: Retrolabyrinthine or retrosigmoid vestibular neurectomy: Indications. Am J Otol 8: 414-418, 1987

18. Silverstein $\mathrm{H}$, Norrell $\mathrm{H}$, Smouha $\mathrm{E}$, et al: Combined retrolabretrosigmoid vestibular neurectomy: An evolution in approach. Am J Otol 10:166-169, 1989

The authors would like to thank Beth Schiffner and Sherry Depue for their technical assistance, and also Beth Lin and Patricia Terrill for their help and critical review of the manuscript.

\section{REVIEWER'S COMMENTS}

I have appreciated very much the paper "Microsurgical Posterior Fossa Vestibular Neurectomy: An Evolution in Technique" by Herbert Silverstein, M.D., and Horace Norrell, M.D. The authors have developed a very interesting concept and alternative technique for sectioning of the vestibular nerve in cases of Meniere's disease. They speak of vestibular neurectomy; however, they do single neurotomy in the three technical variations of "posterior fossa vestibular neurectomy." I'd appreciate their comments on the possibility of nerve regeneration when the vestibular ganglion is left behind the cutting point of the vestibular nerve.

The authors state that in $25 \%$ of cases they should open the internal auditory meatus (IAM) from behind to cut properly the nerve within the IAM. They have observed, however, that this detail results in a chronic and severe headache in $50 \%$ of the patients, persistent for 2 years or more.

When they comment on the operative approaches for vestibular neurectomy, they refer to middle fossa vestibular neurectomy as a technically difficult and formidable procedure with difficult anatomic landmarks and high risk of complications. In our institution we have used the middle fossa vestibular neurectomy for Meniere's disease for the last 16 years. We do not agree that it represents a "formidable" procedure and we do not believe that there are more difficult anatomic landmarks than in other proposed approaches. In the 16 years we have operated on 130 cases of Meniere's disease by the middle fossa vestibular neurectomy approach. The main advantages of this approach, originally proposed by House and modified by Fisch, are the easy identification and 
dissection of the nerves in the lateral half of the IAM and the complete visualization of the entire internal meatus for inspection for disease undetected preoperatively. On the other hand, in these series, the main symptom, vertigo, has been relieved in $92 \%$ of the operated cases. At the same time, we had no cases of deaths or meningitis or liquoric fistulas requiring revision or headache.

The authors are to be congratulated for the concept, persistence, and continuous perfecting of their interesting technique.

Decio Castro, M.D. 